\title{
Doxycycline improves clinical outcomes during cystic fibrosis exacerbations
}

\author{
Xin Xu $\mathrm{u}^{1,2,3,4,5}$, Tarek Abdalla ${ }^{1,2}$, Preston E. Bratcher ${ }^{1,2}$, Patricia L. Jackson ${ }^{1,2,3,4,5}$, \\ Gina Sabbatini ${ }^{6}$, J. Michael Wells ${ }^{1,2,3,4,5}$, Xiang-Yang Lou ${ }^{1,7}$, Rebecca Quinn ${ }^{8}$, \\ J. Edwin Blalock ${ }^{1,2,3,4,9}$, J. P. Clancy ${ }^{10}$ and Amit Gaggar ${ }^{1,2,3,4,5,9}$
}

Affiliations: ${ }^{1}$ Dept of Medicine, Division of Pulmonary, Allergy, and Critical Care, The University of Alabama at Birmingham, Birmingham, AL, USA. ${ }^{2}$ Program in Protease and Matrix Biology, The University of Alabama at Birmingham, Birmingham, AL, USA. ${ }^{3}$ Gregory Fleming James Cystic Fibrosis Research Center, The University of Alabama at Birmingham, Birmingham, AL, USA. ${ }^{4}$ Lung Health Center, The University of Alabama at Birmingham, Birmingham, AL, USA. ${ }^{5}$ Medical Service at Birmingham VA Medical Center, Birmingham, AL, USA. ${ }^{6}$ Physician Assistant Studies Program, Christian Brothers University, Memphis, TN, USA. ${ }^{7}$ Biostatistics Program, Department of Pediatrics, University of Arkansas for Medical Sciences, Little Rock, AR, USA. ${ }^{8}$ Dept of Pharmacy, The University of Alabama at Birmingham, Birmingham, AL, USA. ${ }^{9}$ Dept of Cell, Developmental, and Integrative Biology, The University of Alabama at Birmingham, Birmingham, AL, USA. ${ }^{10}$ Cincinnati Children's Hospital Medical Center and The University of Cincinnati, Cincinnati, OH, USA.

Correspondence: Amit Gaggar, Dept of Medicine, University of Alabama at Birmingham, 422 Tinsley Harrison Tower, Birmingham, AL 35294, USA. E-mail: agaggarQuabmc.edu

@ERSpublications

Doxycycline improved protease/antiprotease imbalance and clinical end-points during CF acute pulmonary exacerbation http://ow.ly/lF9p308Ov6i

Cite this article as: $\mathrm{Xu} \mathrm{X}, \mathrm{Abdalla} \mathrm{T}$, Bratcher PE, et al. Doxycycline improves clinical outcomes during cystic fibrosis exacerbations. Eur Respir J 2017; 49: 1601102 [https://doi.org/10.1183/13993003.01102-2016].

ABSTRACT Matrix metalloprotease-9 (MMP-9) plays a role in progression of cystic fibrosis, and doxycycline can reduce MMP-9 in vitro. Here, we explore the effect of doxycycline during cystic fibrosis exacerbation treatment on MMP-9 related readouts and clinical end-points.

This randomised, double-blind, placebo-controlled study enrolled hospitalised patients with cystic fibrosis undergoing exacerbation. In total, 20 participants were given doxycycline and 19 participants were given placebo over an 8-day period during hospitalisation. Biospecimens were collected at the beginning and the end of the study period. Primary end-points were total MMP-9 levels in the sputum and safety/ tolerability. Secondary end-points included change in lung function, time to next exacerbation, and markers of MMP-9-related protease activity (active MMP-9 and TIMP-1). Nonparametric testing was used for within-group and between-group analyses.

Doxycycline was well tolerated, with no treatment discontinuations or serious adverse events. Doxycycline reduced total sputum MMP-9 levels by $63.2 \%(\mathrm{p}<0.05)$, and was also associated with a $56.5 \%$ reduction in active MMP-9 levels $(\mathrm{p}<0.05)$, a 1.6-fold increase in sputum TIMP-1 $(\mathrm{p}<0.05)$, improvement in forced expiratory volume in $1 \mathrm{~s}(\mathrm{p}<0.05)$, and an increase in time to next exacerbation $(\mathrm{p}<0.01)$.

Adjunctive use of doxycycline improved dysregulated MMP-9 levels in sputum, along with biomarkers consistent with a reduced proteolytic pulmonary environment. Improvement in clinical outcome measures suggests an important therapeutic benefit of doxycycline for individuals with cystic fibrosis.

This article has supplementary material available from erj.ersjournals.com

Received: June 012016 | Accepted after revision: Dec 192016

This study is registered at ClinicalTrials.gov with identifier number NCT01112059.

Copyright @ERS 2017 


\section{Introduction}

Cystic fibrosis (CF) is the most common inherited genetic disorder in Caucasians worldwide [1]. It is due to loss-of-function mutations in the cystic fibrosis transmembrane conductance regulator (CFTR), which disrupts normal epithelial cell function [2]. The most prominent manifestations are observed in the lungs, where the loss of mucociliary clearance in the airway leads to mucostasis, infection and ongoing inflammation [3]. Eventually, this ongoing inflammation promotes extracellular matrix remodelling and bronchiectasis in the CF airways, leading to impaired gas exchange, increased bacterial colonisation and increased disease-related mortality [4]. The progressive lung remodelling observed in CF lung disease starts early in life [4], and recent animal studies have suggested that these changes are important in both the small and large airways [5]. Despite the direct impact of this airway remodelling on disease outcome, there are currently no specific anti-fibrotic treatments and only one anti-protease therapy within the current CF Foundation drug development pipeline (https://tools.cff.org/research/drugdevelopmentpipeline). Indeed, the lung remodelling of extracellular matrix products such as collagen and elastin lead to loss of connective tissue integrity of both the airways and parenchyma, and to loss of lung elastic recoil [6]. These effects contribute to a gradual worsening of the ventilation/perfusion mismatch in the CF lung [7], leading to hypoxaemia and loss of lung function.

In addition to the steady decline in lung health, the disease course is characterised by progressive decline in lung function with worsening pulmonary symptoms and episodic loss of airflow [8]. These intermittent episodes are termed CF acute pulmonary exacerbations (APE), and they strongly contribute to disease-associated morbidity and mortality [9]. During APE episodes, the CF airways exhibit increased bacterial burden and a neutrophilic inflammatory response with prominent protease activation [10]. Current treatment of APEs is directed at improving mucus clearance and treating the airway infection with intravenous antibiotics [9].

A number of proteases have emerged as important contributors in lung remodelling and associated inflammation in the CF airways, with most of the attention being focused on elevated levels and activity of human neutrophil elastase (NE) [11]. Recently, a different protease, matrix metalloprotease-9 (MMP-9), has been shown to be differentially elevated in the airway secretions of patients with CF lung disease [12]. MMP-9 is a metalloprotease that is released from neutrophils, macrophages and epithelial cells in the airway [13] as a pro-enzyme, and is subsequently activated by loss of its pro-domain, thereby leading to an active enzyme and a separate pool of pro-enzyme. Our research has demonstrated that during acute CF inpatient exacerbations, both airway MMP-9 levels and activity are significantly increased relative to the natural occurring MMP inhibitor, tissue inhibitor of metalloprotease-1 (TIMP-1), and this imbalance represents a three-fold increase relative to clinically stable patients with CF [12]. As a result, this proteaseantiprotease imbalance can augment airway remodelling, affect airway immunity and modulate airway host-pathogen interactions during the peri-exacerbation period [14]. For example, recently our group described increased levels of an MMP-9-generated extracellular matrix fragment, acetylated prolineglycine-proline (Ac-PGP), in the CF airway as a critical modulator of neutrophilic inflammation [15]. As a result of the potential downstream impact of this protease dysregulation, there is significant interest in developing novel anti-MMP therapeutics, although these efforts have been largely unsuccessful, mainly due to poorly tolerated side-effect profiles (i.e. arthralgias with hydroxamate inhibitors) or lack of appropriate drug delivery in the lungs [16].

Doxycycline is a tetracycline antibiotic widely used in the treatment of infections caused by Gram-positive organisms. Previous research has demonstrated that doxycycline harbours anti-MMP activity, with initial reports suggesting that it can serve as a small molecule inhibitor of MMP-9 [17, 18]. However, subsequent literature has suggested that doxycycline also affects MMP-9 transcription [19, 20]. These results provide a scientific rationale to examine doxycycline as a therapeutic directed to dysregulated MMP-9 in CF lung disease. Therefore, we conducted a randomised, double-blind, placebo-controlled trial to determine if doxycycline was well tolerated and could alter the MMP-9 activity in the sputum of patients with CF during the treatment of inpatient CF APE. In addition to these primary objectives, secondary endpoints included biomarkers related to the proteolytic environment, inflammation and clinical parameters (lung

Support statement: These studies were funded in part by grants from the NIH (HL07783, HL090999 and HL087824 to J.E. Blalock; HL102371 to A. Gaggar; K08HL123940 to J.M. Wells), Cystic Fibrosis Foundation Therapeutics (GAGGARA0 to A. Gaggar), the Veterans Administration (1 I01 BX001756 to A. Gaggar) and the Ismail Moustapha Scholar Fund (to A. Gaggar). The research reported in this publication was supported by the NIH and the Family Smoking Prevention and Tobacco Control Act. The content is solely the responsibility of the authors and does not necessarily represent the official views of the NIH or the Food and Drug Administration. Funding information for this article has been deposited with the Open Funder Registry.

Conflict of interest: Disclosures can be found alongside this article at erj.ersjournals.com 
function, length of hospital stay and time to next exacerbation). Therefore, this study design provides mechanistic insight for the effect of doxycyline on airway biology, and presents evidence regarding this agent as a potential therapeutic in chronic lung disease with protease-antiprotease imbalance.

\section{Methods}

\section{Patients}

All participants had been diagnosed with CF based on routine diagnostic criteria, including two sweat $\mathrm{Cl}^{-}$values $>60 \mathrm{mM}$ and a minimum of two clinical features consistent with the diagnosis [2]. Inclusion criteria included diagnosis of $\mathrm{CF}$, age $>19$ years, colonisation with Pseudomonas aeruginosa, and undergoing APE requiring inpatient care. Notable exclusion criteria included allergy or sensitivity to doxycycline or tetracycline; concurrent or recent (within 28 days of enrolment) use of corticosteroids ( prednisone $>5 \mathrm{mg}^{-\mathrm{day}^{-1}}$ ); inability to spontaneously produce sputum; acute respiratory failure requiring the use of invasive or noninvasive ventilation, chronic liver or renal disease; pregnancy, colonisation by Burkholderia species and active colonisation by mycobacterial species. Patients were screened at the time of inpatient admission, and informed written consent was obtained from all participants included in the study. During the course of the study, a quarterly data safety monitoring board meeting was conducted to assess any safety considerations and provide feedback to the principal investigator regarding study conduct.

\section{Study design}

This study was a single-centre, phase II, randomised, double-blind, placebo-controlled trial for adult patients with CF, performed at the University of Alabama at Birmingham Hospital (UAB) in Birmingham, AL, USA (ClinicalTrials.gov NCT01112059). Subjects were recruited by the principal investigator or study coordinators from a CF inpatient population from June 2010 to June 2012. All participants met Fuchs criteria [8] to be enrolled in the study. Consenting patients were randomly allocated in equal numbers to receive doxycycline $100 \mathrm{mg}$ orally twice daily or an identical-appearing placebo for 8 days during the course of hospitalisation for treatment of APE. Subjects remained on all CF chronic care medications and standard inpatient exacerbation therapy (intravenous antibiotics and increased airway clearance) throughout the course of the study. The study pharmacist filled the prescriptions by computer-generated random assignment in blocks of four participants, and had sole access to the randomisation code. All participants were given either doxycycline or placebo by nursing staff on the CF inpatient service. The drugs were in blister packs, and their appropriate use was validated by both research coordinator and research pharmacist daily. No variance from protocol was reported throughout the study.

Of note, standard patient care for participants and decision for discharge were conducted by CF care physicians who were blinded to study assignment. Spirometry was conducted at beginning and end of hospitalisation, using American Thoracic Society guidelines [21]. Sputum bacteriology and serum C-reactive protein (CRP) were collected at the beginning of hospitalisation as part of standard care. Brief histories and physical examinations were conducted for all participants at the beginning of hospitalisation, at hospitalisation day 8 , and at time of discharge. Additional sputum and blood were collected at baseline before the treatment, and at patient discharge for trial-specific readouts (figure 1a). Of note, only 36 of the 39 analysable participants were able to provide sputum at both time points, and 38 of the 39 analysable participants were able to provide blood at both time points. Only participants with complete sets of data were analysed for a given outcome measure. All 39 participants were analysed for clinical readouts such as lung function, hospital stay and time to next exacerbation. For the time to next exacerbation metric, we prospectively followed the clinic visits of study participants with CF providers at UAB, identifying evidence of first exacerbation any point after discharge at these visits by documented Fuchs criteria. We conservatively determined an exacerbation only when appropriate Fuchs criteria were met and parental antibiotics were instituted.

The primary study end-points were total MMP-9 levels in the sputum and safety/tolerability of the treatment. The secondary study end-points included change in active MMP-9, lung function, length of hospitalisation stay, time to next exacerbation, markers of MMP-9 related protease activity and markers of inflammation (i.e. NE, CRP, pro-inflammatory cytokines). All patients had a 1-month follow-up call after discharge from hospital.

\section{Biochemical assays}

Serum and sputum were processed as described in the online data supplement. MMP-9 specific ELISA and activity, TIMP-1 ELISA, NE ELISA assays, cytokine analyses and Ac-PGP peptide detection were conducted as described in the online data supplement.

\section{Statistical testing}

Based on our prior work [12], we estimated that a sample of 20 patients per group would provide the trial with a power of $80 \%$ and $\alpha=0.05$ to show a $45 \%$ reduction in total MMP- 9 in the doxycycline-treated 
a)

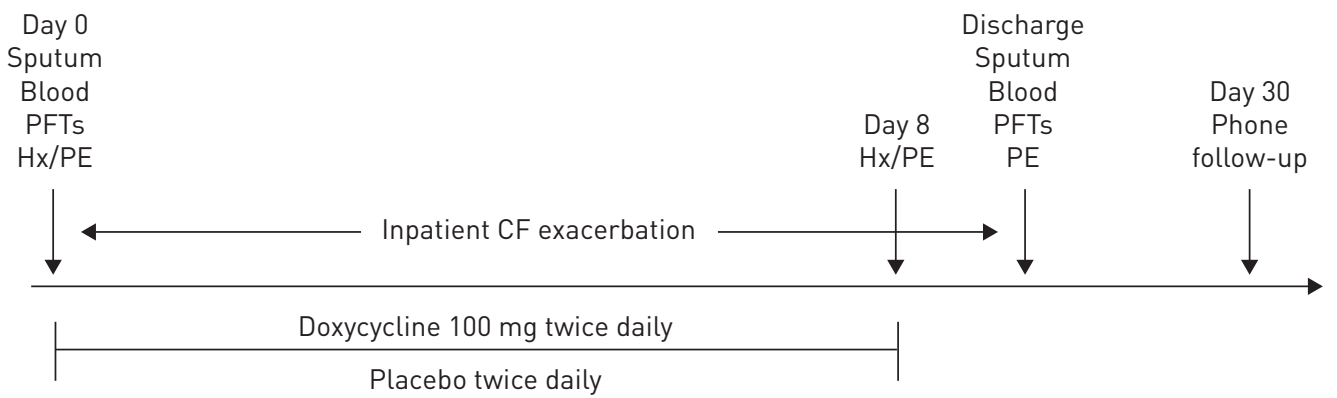

b)

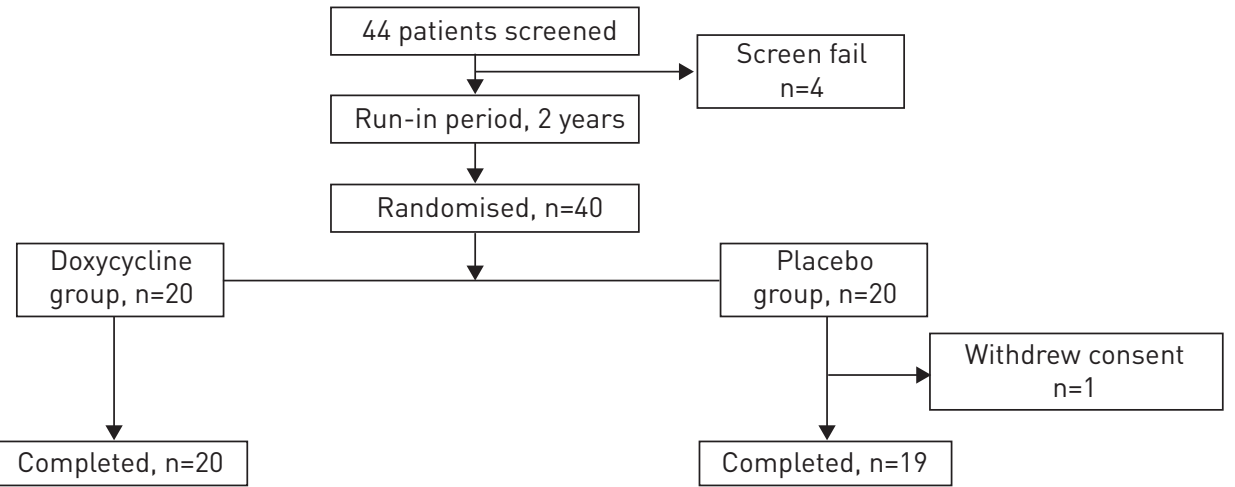

FIGURE 1 Clinical trial design and CONSORT diagram. a) Doxycycline as adjunctive treatment for an inpatient cystic fibrosis (CF) exacerbation trial design: all inpatients with CF with exacerbation were asked for consent within $24 \mathrm{~h}$ of admission and treated for 8 days. Information on biomarkers and patients' physical state were collected prior to drug treatment and discharge. Primary end-points included safety/tolerability and total matrix metalloproteinase (MMP)-9 activity. Secondary end-points include inflammatory markers and change in lung function. All patients were followed up by phone 30 days later after discharge. $\mathrm{Hx}$ : medical history; PE: physical examination; PFTs: pulmonary function tests. b) CONSORT diagram for this clinical trial: 44 patients were screened for the study; of these, 40 participants were deemed eligible and were randomised to one of two study groups. One subject withdrew consent during the course of the study, thus 39 participants $(n=20$ in the doxycycline group and $n=19$ in the placebo groupl underwent complete follow-up and were included in the analyses.

group after 8 days of therapy. Descriptive statistics including mean \pm SD were calculated for all quantitative measures and are displayed in figures (unless otherwise stated in figure legend). Demographic and diagnostic comparisons between doxycycline-treated and placebo control participants were performed using robust nonparametric statistical testing to avoid potential bias due to the violation of normality assumption for end-points. Comparisons between pre-treatment and post-treatment measurements for biomarkers and lung function were performed using the Wilcoxon signed rank test for within-treatment group analysis, and the degree of change per group were compared across groups by Mann-Whitney statistic. Kaplan-Meier analysis with log-rank test was used to determine whether patients treated with doxycycline and placebo differed significantly in time to next exacerbation. The treatment effect was further estimated as hazard ratio (HR) with 95\% confidence interval computed by a Cox proportional hazard model with adjustment for race, sex and age as covariates. Variables showing a univariate association with change in forced expiratory volume in $1 \mathrm{~s}\left(\mathrm{FEV}_{1}\right)(\mathrm{p}<0.05)$ were included in a stepwise, backwards, multiple linear regression model to identify independent association between clinical factors, sputum biomarkers and percentage change in $\mathrm{FEV}_{1}$ over the study period. Statistical tests were two-sided, and $\mathrm{p}<0.05$ was deemed statistically significant. Calculations were made using Instat software (GraphPad, version 5.0), SPSS Statistics Software (version 20.0, IBM Corporation), or SAS (version 9.4, SAS Institute).

\section{Study approval}

Human participants provided written informed consent. The study protocol was approved by the institutional review board at UAB (protocol F081024004).

\section{Results}

Patient demographics and safety

The baseline characteristics of the participants are described in table 1, with both groups being closely matched. Overall, both groups exhibited baseline moderate to severe airway obstruction, with stable outpatient 


\begin{tabular}{|c|c|c|c|}
\hline Baseline variable & Doxycycline & Placebo & p-value \\
\hline Subjects $\mathrm{n}$ & 20 & 19 & \\
\hline Age years & $29.1 \pm 8.9$ & $27.3 \pm 9.4$ & 0.55 \\
\hline \multicolumn{4}{|l|}{ Ethnicity } \\
\hline White & $18(90 \%)$ & 17 (89\%) & \multirow[t]{2}{*}{0.98} \\
\hline Black & $2(10 \%)$ & $2(11 \%)$ & \\
\hline \multicolumn{4}{|l|}{ Sex } \\
\hline Male & $12(60 \%)$ & $13(68.4 \%)$ & \multirow[t]{2}{*}{0.60} \\
\hline Female & $8(40 \%)$ & $6(31.6 \%)$ & \\
\hline \multicolumn{4}{|l|}{ Mutation } \\
\hline Delta F508 homozygous & $8(40 \%)$ & $9(47 \%)$ & 0.66 \\
\hline Delta F508 heterozygous & $8(40 \%)$ & $8(42 \%)$ & 0.91 \\
\hline Other & $4(20 \%)$ & $2(11 \%)$ & 0.43 \\
\hline \multicolumn{4}{|l|}{ Lung function } \\
\hline Admission $\mathrm{FEV}_{1} \mathrm{~L}$ & $1.50 \pm 0.71$ & $1.36 \pm 0.67$ & 0.53 \\
\hline Admission $\mathrm{FEV}_{1} \%$ pred & $47.4 \% \pm 19.6$ & $42.4 \% \pm 11.6$ & 0.24 \\
\hline Admission FVC L & $2.55 \pm 0.97$ & $2.34 \pm 1.00$ & 0.51 \\
\hline Admission FVC \% pred & $56.8 \% \pm 13.1$ & $49.0 \% \pm 15.8$ & 0.16 \\
\hline Admission $\mathrm{FEF}_{25-75 \%} \%$ & $33.4 \% \pm 28.6$ & $16.4 \% \pm 6.2$ & 0.24 \\
\hline \multicolumn{4}{|l|}{ CF-related conditions } \\
\hline Pancreatic insufficiency & $19(95 \%)$ & $19(100 \%)$ & 0.36 \\
\hline CFRD & $6(30 \%)$ & $8(42 \%)$ & 0.45 \\
\hline \multicolumn{4}{|l|}{ Chronic care medications } \\
\hline Azithromycin & $18(90.0 \%)$ & $15(79 \%)$ & 0.36 \\
\hline Hypertonic saline & $7(35 \%)$ & $9(47 \%)$ & 0.45 \\
\hline Inhaled tobramycin & $11(55 \%)$ & $9(47 \%)$ & 0.65 \\
\hline Dornase- $\alpha$ & $18(90 \%)$ & $18(95 \%)$ & 0.61 \\
\hline Inhaled colistin & $7(35 \%)$ & $9(47 \%)$ & 0.45 \\
\hline Inhaled aztreonam & $6(30 \%)$ & $3(16 \%)$ & 0.31 \\
\hline \multicolumn{4}{|l|}{ Sputum bacteriology } \\
\hline Pseudomonas aeruginosa & $20(100 \%)$ & $19(100 \%)$ & 1.0 \\
\hline Mucoid strain only & $8(40 \%)$ & $4(21 \%)$ & 0.21 \\
\hline Nonmucoid strain only & $3(15 \%)$ & $7(37 \%)$ & 0.13 \\
\hline Mucoid+nonmucoid strain & $9(45 \%)$ & $8(42 \%)$ & 0.87 \\
\hline Staphylococcus aureus & $9(45 \%)$ & $13(67 \%)$ & 0.15 \\
\hline Methicillin-resistant & $7(35 \%)$ & $11(57 \%)$ & 0.16 \\
\hline Methicillin-sensitive & $2(10 \%)$ & $2(10.5 \%)$ & 0.98 \\
\hline \multicolumn{4}{|c|}{ Intravenous antibiotics in hospital } \\
\hline Aminoglycoside $+\beta$-lactam & $20(100 \%)$ & $19(100 \%)$ & 1.0 \\
\hline Vancomycin or linezolid & $7(35 \%)$ & $11(57 \%)$ & 0.16 \\
\hline Body mass index & $19.9 \pm 3.9$ & $20.2 \pm 2.7$ & 0.67 \\
\hline Serum CRP & $56.1 \pm 63$ & $37.8 \pm 40.1$ & 0.35 \\
\hline
\end{tabular}

Continuous variables reported as mean $\pm \mathrm{SD}$. $\mathrm{FEV}_{1}$ : forced expiratory volume in $1 \mathrm{~s}$; FVC: forced vital capacity; $\mathrm{FEF}_{25-75}$ : forced expiratory flow at $25-75 \%$ of FVC; CF: cystic fibrosis; CFRD: cystic fibrosis-related diabetes; CRP: C-reactive protein.

$\mathrm{FEV}_{1}$ of $45.8 \pm 16.3 \%$ and $50.2 \pm 17.7 \%$ for the placebo and doxycycline groups, respectively ( $\mathrm{p}=0.58$ ). In addition, both groups had similar average exacerbation frequency in the year prior to hospitalisation (2.9 exacerbations in placebo group versus 2.8 exacerbations in the doxycycline group; $\mathrm{p}=0.61$ ).

Both groups had comparable sputum bacteriology (methicillin-sensitive Staphylococcus aureus (MSSA) and Pseudomonas aeruginosa), although the placebo group had a nonsignificant higher incidence of methicillin-resistant S. aureus (MRSA). Of note, one patient each in the doxycycline group and placebo group had a positive culture for Stenotrophomonas maltophilia, both of which were sensitive to doxycycline. Figure 1a describes the schema of the clinical trial with notations of sputum and blood collection. During hospitalisation, both groups were treated with standard care including intravenous aminoglycoside plus beta-lactam antimicrobials, as well as enhanced airway clearance via chest percussion therapy twice daily. Patients with MRSA were additionally treated with either intravenous vancomycin or oral linezolid. Baseline CF medications continued for all participants during hospitalisation.

The CONSORT diagram for this trial is shown in figure 1b. Overall, four individuals were deemed screen failures (two due to lack of sputum production, one due to active haemoptysis and one due to initiation of 
i.v. steroids early in their hospital course). The remaining 40 participants were randomised to one of two groups using a block randomisation schema via the UAB Research Pharmacy. One subject in the placebo group withdrew consent during the course of the study. All 39 participants completing the study were alive at 30 days after hospital discharge. Of note, no significant adverse events were noted during the study period, and 10 adverse events were observed (five per group), all of which were self-limiting. Only one of the adverse events in the doxycycline group was judged as possibly related to the study drug (tingling of lips for 1 day, self-resolving).

\section{Airway MMP-9 levels and activity are reduced by doxycycline}

At baseline, each study population demonstrated comparable elevations in total MMP-9 (pro-MMP-9 plus active MMP-9) levels, with over $90 \%$ of the measureable MMP-9 being active. At the end of hospitalisation, total MMP-9 activity showed a significant decrease in the doxycycline ( $\mathrm{p}=0.01$ ) (figure 2a) but not in the placebo group (figure $2 \mathrm{~b}$ ). In addition, there was a 56.5\% reduction in active MMP-9 levels in the doxycycline group $(\mathrm{p}<0.05)$ (figure $2 \mathrm{c}$ ), suggesting that doxycycline affected airway MMP-9 protein and enzymatic activity. Serum levels of MMP-9 were also lower than sputum levels in both treatment groups, and were relatively unchanged after inpatient therapy (figure S1).

\section{Doxycycline attenuates the pro-proteolytic CF environment}

Previous studies have highlighted that the pro-proteolytic environment in the CF airways has a significant impact on ongoing protease-antiprotease imbalance, extracellular matrix remodelling and airway inflammation [11, 14]. To address this balance, we quantified sputum TIMP-1 levels, and observed that although baseline levels were comparable between the two groups, the doxycycline-treated group had a 1.6-fold increase over the course of treatment compared with the placebo group $(\mathrm{p}<0.05$, figure $3 a)$. When total levels were examined relative to TIMP-1, the doxycycline-treated group had a $>70 \%$ reduction (figure 3c), demonstrating an improved balance between this critical protease-antiprotease complex in the CF airway.

Sputum Ac-PGP levels were reduced in the doxycycline-treated group (figure 3e) but not in the placebo group (figure 3f) post-treatment, reinforcing the observation of a reduced proteolytic environment. No difference was observed in NE levels between the two groups over the course of the study (figure S2a), suggesting that this antiprotease effect was specific for MMP activity. Levels of CRP dropped in both groups, but there was no difference observed with either placebo or doxycycline treatment (figure S2b).

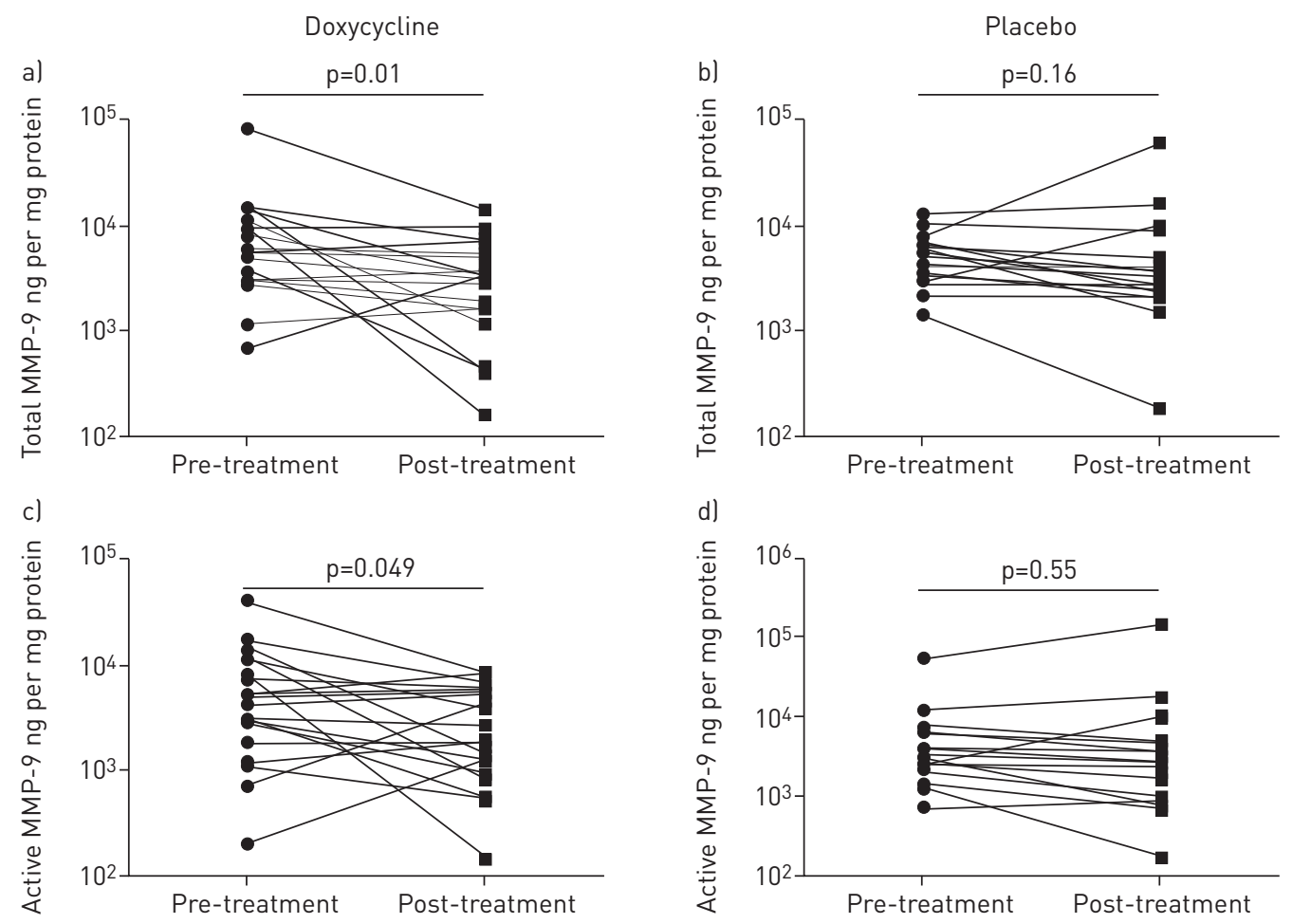

FIGURE 2 Neutralisation of airway matrix metalloproteinase (MMP)-9 with doxycycline treatment: sputum total MMP-9 $(a, b)$ and active MMP-9 (c, d) were quantified and standardised to total protein, $n=19$ for doxycycline and $n=17$ for placebo. Data displayed for each sample pre-versus post-therapy. 


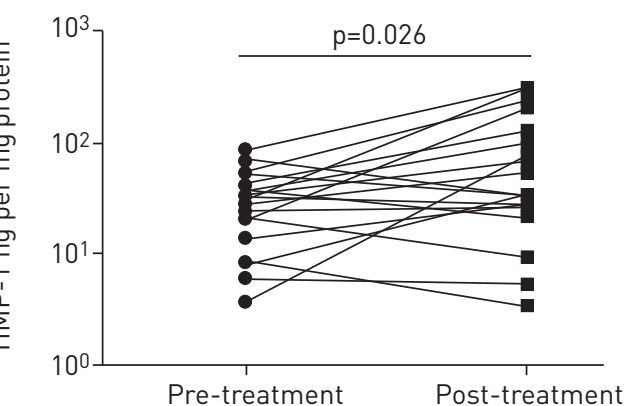

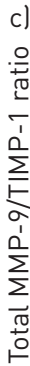



FIGURE 3 Restoration of protease-antiprotease balance and reduction of extracellular matrix breakdown markers in cystic fibrosis airways by doxycycline treatment: sputum tissue inhibitor of metalloproteinase (TIMP)-1 (a, b) was quantified and compared with total matrix metalloproteinase (MM)P-9 (c, d); extracellular matrix breakdown marker sputum acetylated proline-glycine-proline (Ac-PGP) (e, f) was measured by electrospray ionisation-liquid chromatography tandem mass spectrometry and standardised to total protein. $\mathrm{n}=19$ for doxycycline and $\mathrm{n}=17$ for placebo. Data displayed for each sample pre-versus post-therapy.

Additionally, no differences between the placebo and doxycycline groups were observed for classic early response pro-inflammatory cytokines (table 2).

\section{Doxycycline improves clinical outcome measures during CF inpatient exacerbation}

In conjunction with biochemical changes observed during doxycycline treatment, we also examined the impact of doxycycline on clinical outcomes during CF APE. The forced vital capacity (FVC) total volume and \% predicted was improved in both treatment groups over the course of exacerbation, but was not statistically different between the two groups (figure S3a and b). These results paralleled changes in forced expiratory flow at $25-75 \%$ of $\mathrm{FVC}\left(\mathrm{FEF}_{25-75}\right)$ (figure $\mathrm{S3c}$ ). Although the $\mathrm{FEV}_{1}$ was improved in both treatment groups (figure $4 \mathrm{a}$ and $\mathrm{b}$ ), the change in both total volume (figure $4 \mathrm{c}$ ) and \% pred (figure $4 \mathrm{~d}$ ) was significantly higher in the doxycycline-treated cohort compared with the placebo cohort. The group treated with doxycycline had a reduction in total hospital days compared with the placebo group (12.7 versus 14.2 days respectively; $\mathrm{p}=0.17$ ). We also observed that the doxycycline group had fewer total i.v. antibiotic days relative to the placebo group (13.4 versus 15.3 days; $\mathrm{p}=0.064$ ). Finally, we found a significant improvement in time to next exacerbation in the doxycycline group compared with the placebo group (figure 5) $(\mathrm{p}<0.01$ by log-rank test), with an adjusted HR of 0.289 (95\% CI $0.128-0.633 ; \mathrm{p}<0.01$ ). There was no observed difference in MRSA-positive versus MRSA-negative participants (figure S3d). 
TABLE 2 Sputum cytokine levels before and after treatment $\left(\mathrm{pg} \cdot \mathrm{mL}^{-1}\right.$ )



Data are presented as mean \pm SD. IL: interleukin; MIP: macrophage inflammatory protein; TNF: tumour necrosis factor.

\section{Doxycycline treatment is independently associated with lung function improvement}

Age, race, sex, genotype, body mass index, Pseudomonas aeruginosa (mucoid and nonmucoid strains) and Staphylococcus (MRSA and MSSA) infection were not associated with improvement in $\mathrm{FEV}_{1} \%$ pred in univariate analyses. Among the biomarkers studied, change in $\mathrm{FEV}_{1} \%$ pred was correlated with \% change in sputum MMP-9 activity standardised to TIMP-1 $(\rho=-0.34, p<0.05)$, but not with changes in other biomarkers in blood or sputum. In a multiple linear regression model adjusting for baseline $\mathrm{FEV}_{1}(\%)$, treatment allocation, and \% change in MMP-9 activity/TIMP-1, only doxycycline therapy was independently associated with a change in $\mathrm{FEV}_{1} \%$ during the study period (standardised $\beta=0.34, \mathrm{p}<0.05$ ).

\section{Discussion}

Treatment of CF has evolved significantly over the past 20 years, with the development of novel antibacterial, anti-inflammatory, airway hydration and most recently CFTR modulator therapies [1]. However, despite these substantial therapeutic gains, the ongoing airway remodelling with bronchiectasis remains a critical challenge in the treatment of progressive CF lung disease, a process that is augmented
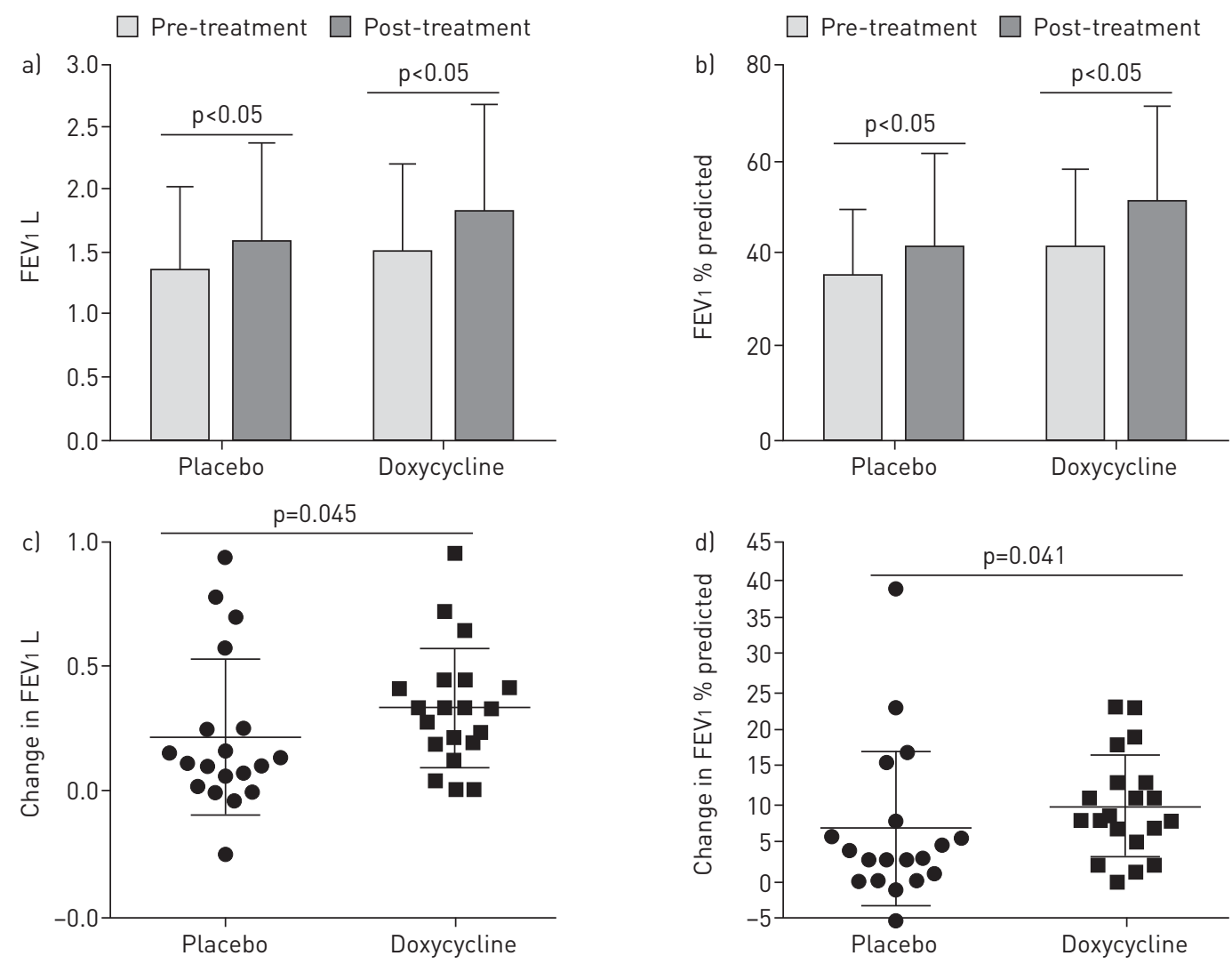

FIGURE 4 Doxycycline improves clinical parameters during cystic fibrosis exacerbation: absolute forced expiratory volume in $1 \mathrm{~s}$ (FEV1) (a) and $\mathrm{FEV}_{1} \%$ predicted (b) before and after exacerbation, along with the difference for each individual (c and d, respectively), are displayed for each treatment group. $n=20$ for doxycycline and $n=19$ for placebo. 


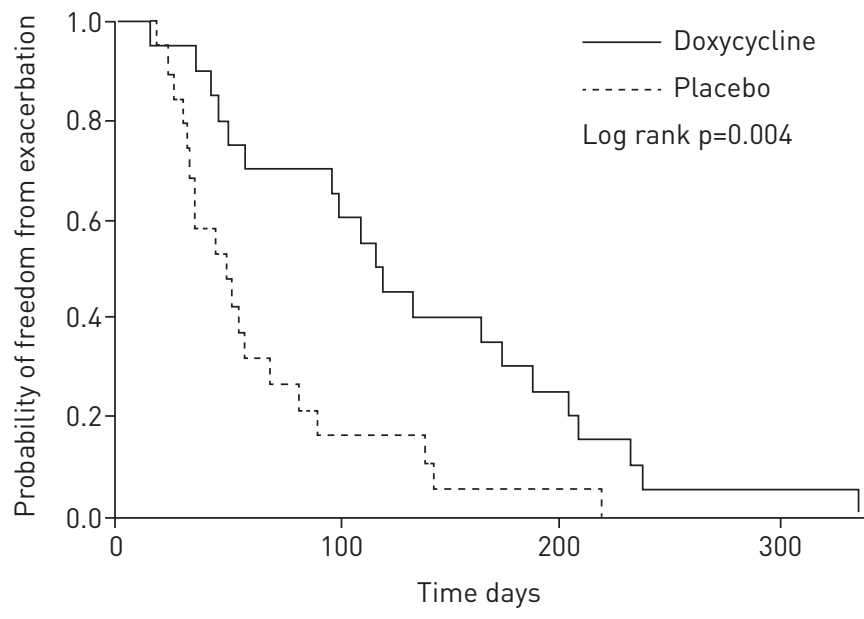

FIGURE 5 Doxycycline improves time to next exacerbation during cystic fibrosis exacerbation: Kaplan-Meier nonexacerbation curves with log-rank test are shown for the difference in time to next exacerbation associated with doxycycline therapy (solid line) compared with placebo (dotted line). $n=20$ for doxycycline and $\mathrm{n}=19$ for placebo.

during APE. The current study represents one of the largest interventional clinical trial targeted specifically at CF APE within the past 20 years [9], illuminating the need for additional randomised controlled studies in this population. Pulmonary exacerbations are critical periods in the disease course of $\mathrm{CF}$, with strong predictive relationships with loss of lung function, future APE and mortality $[2,5,6]$. Our study highlights the utilisation of a very well-tolerated agent as a proof in concept of the potential benefits of targeting elevated protease activity in CF lung disease.

The two study cohorts were well matched for demographics and clinical features of CF disease. One notable difference between the populations was the difference in concurrent $S$. aureus colonisation, with all MRSA and MSSA colonised participants harbouring doxycycline-sensitive S. aureus by in vitro testing. MRSA has been associated with worse survival [22], suggesting that this may be an important confounder to the current study. However, as all participants with MRSA were treated with MRSA-specific therapy (linezolid and vancomycin), the potential impact of doxycycline on MRSA appears minimal. In addition, univariate analysis did not demonstrate an association between change in lung function and MRSA colonisation status. Similarly, subgroup analysis of time to next exacerbation did not demonstrate differences with MRSA colonisation status. In total, these findings strongly suggest that the beneficial outcomes of doxycycline are independent of antimicrobial effects on S. aureus. However, future studies should include MSSA and MRSA block randomisation and responder analysis based on S. aureus antimicrobial susceptibility.

Our previous data has demonstrated that MMP-9 levels are disproportionately increased in the CF airway (compared with circulating levels), with the majority of sputum MMP-9 in an active state and very little as pro-enzyme during APE [12]. These findings emphasise the importance that a systemically delivered medication must reach sufficient levels to be detectable in airway secretions. A recent study has demonstrated that $200 \mathrm{mg}$ per day dose of oral doxycycline allows for a concentration of doxycycline in the sputum of individuals with CF [23] that is capable of neutralising MMP-9 activity [24].

Our results clearly demonstrate a neutralisation of sputum MMP-9 in the doxycycline treatment group in the context of receiving a variety of chronic care medications, including azithromycin, an agent with potential anti-inflammatory activities [25]. In accordance with changes in MMP-9 levels and activity in the doxycycline-treated population, we observed improvements in both TIMP-1 and Ac-PGP levels in the sputum. TIMP-1 has previously been reported to be increased with doxycycline in a murine model of inflammation [26], but our study is the first to demonstrate this in human disease. These results suggest the possibility of a single drug having an impact on restoration of MMP-9/TIMP-1 balance by both reducing MMP-9 burden and increasing endogenous TIMP-1 levels. In addition, Ac-PGP is an extracellular matrix breakdown product directly related to MMP-9 activity in CF lung disease [27, 28] and therefore, attenuation of MMP-9 in the CF lung may have an impact on airway remodelling [29].

Despite these robust effects on MMP-9, doxycycline did not change CRP levels in the serum or inflammatory cytokine levels in the sputum. Although previous literature utilising in vitro and in vivo models have suggested that doxycycline has an effect on inflammatory cytokine expression [30, 31], clinical trials have also failed to observe these findings $[32,33]$. There was also no difference in change of 


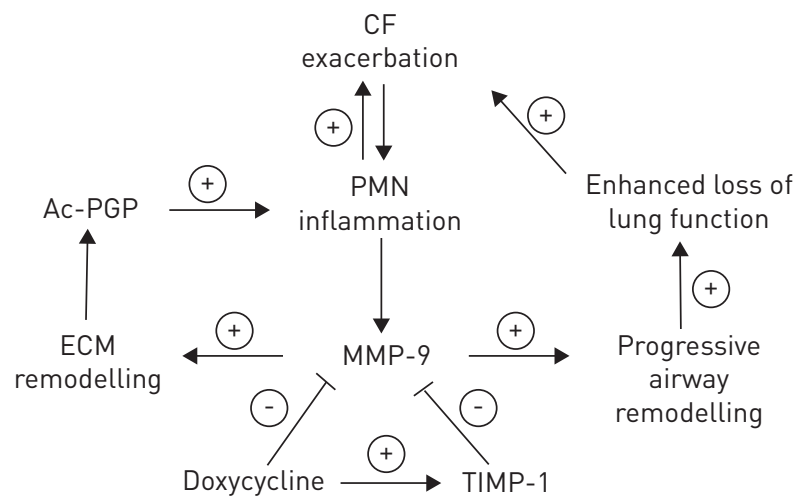

FIGURE 6 Model of doxycycline as a novel adjunctive therapy during cystic fibrosis (CF) exacerbations. CF acute pulmonary exacerbations exhibit increased bacterial burden and a neutrophilic inflammatory response. Matrix metalloproteinase (MMP)-9 is released from polymorphonuclear (PMN) leukocytes, along with other proteases such as neutrophil elastase. In addition to augmenting proteolytic damage to the lung parenchyma, dysregulated MMP-9 activity can break down extracellular matrix (ECM) and enhance acetylated prolineglycine-proline (Ac-PGP) to increase neutrophil influx into the airways. Doxycycline had an impact on restoration of MMP-9/ tissue inhibitor of metalloproteinase (TIMP)-1 balance by both reducing MMP-9 burden and increasing endogenous TIMP-1 levels, attenuating airway remodelling, and improving clinical outcomes such as forced expiratory volume in $1 \mathrm{~s}$ and increased time to next exacerbation.

circulating MMP-9 between the placebo and doxycycline group, likely reflecting the lower levels of systemic MMP-9 compared with the pulmonary compartment.

This study demonstrated a reduction in total hospital days for the doxycycline participants, although this result may have been affected by non-clinical factors such as a patient's ability to complete therapies at home versus hospital. Perhaps the most encouraging findings in this study were the improvement in $\mathrm{FEV}_{1}$ in the doxycycline group relative to the placebo group and the change in time to next exacerbation. The degree of relative improvement in airflow obstruction underscores the importance of protease imbalance in CF lung disease. The degree of improvement in $\mathrm{FEV}_{1}$ in the doxycycline group over the placebo group (approximately $110 \mathrm{~mL}$ and absolute increase of $4.1 \%$ in FEV1\% predicted) was comparable to other pulmonary therapies used for the chronic management of CF, including dornase-alpha, hypertonic saline, azithromycin [34-36] and most recently, ivacaftor/lumacaftor in patients with CF homozygous for the delta F508 mutation [37]. Although it is difficult to draw conclusions in relative efficacy due to the differences in study design across these various agents, they provide a context for the clinical impact of our results. Similarly, it is possible that the improved treatment in protease-antiprotease imbalance modulated by doxycycline positively affected APE resolution, providing a more quiescent post-exacerbation baseline and extending the time until the following exacerbation. Although our single-centre study followed a population of patients with CF exclusively receiving their care at our institution, a future multicentre trial will have to capture all exacerbation events (including those outside of the CF centres) to validate our time to next exacerbation results.

There are several limitations to consider regarding this study. Although the study has a relatively large number of participants undergoing APE, it was a single-centre trial. In addition, although it is quite likely that these clinical findings are mediated by modulating the dysregulated protease activity in the airway, it is possible that these readouts may be mediated by other potential biological effects, such as alterations in airway mucostasis, changes in lung cell signalling or alterations in airway microbiome over this short treatment window. Finally, we did not assess alterations in quality-of-life measures with well-validated questionnaires in this study. Regardless, these findings present a critical study that provides the onus for a multicentre, randomised, double-blind, placebo-controlled trial that can be designed and powered to examine these additional biological mechanisms along with validating the results of this single-centre study.

In conclusion, our study demonstrated MMP-9 neutralisation, tolerability of doxycycline and meaningful clinical improvements during and after APE (figure 6). These results, coupled with an excellent safety profile, minimal financial burden and improvement in the pulmonary proteolytic signature, demonstrate strong potential for doxycycline as a unique therapeutic for treatment of APE in CF lung disease.

\section{Acknowledgements}

The authors would like to thank George (Marty) Solomon and Steven Rowe for their assistance in the organisation of this manuscript, and members of the CF Clinical Research Team at UAB and Nathaniel Weathington for guidance on patient recruitment. We would also like to thank Anuj Gaggar and Eric Sorscher for their critical reading of this manuscript. Most importantly, the authors would like to thank the patients who agreed to enrol in this clinical study. 
Author contributions: conception and design: X. Xu, G. Sabbatini, R. Quinn, J.E. Blalock, J.P. Clancy, A. Gaggar; analysis and interpretation: X. Xu, T. Abdalla, P.E. Bratcher, P.L. Jackson, J.M. Wells, X-Y. Lou; drafting the manuscript for important intellectual content: X. Xu, X-Y. Lou, J.E. Blalock, J.P. Clancy, A. Gaggar.

\section{References}

1 Rowe SM, Borowitz DS, Burns JL, et al. Progress in cystic fibrosis and the CF Therapeutics Development Network. Thorax 2012; 67: 882-890.

Rowe SM, Miller S, Sorscher EJ. Cystic fibrosis. N Engl J Med 2005; 352: 1992-2001.

O’Sullivan BP, Freedman SD. Cystic fibrosis. Lancet 2009; 373: 1891-1904.

4 Regamey N, Jeffery PK, Alton EW, et al. Airway remodelling and its relationship to inflammation in cystic fibrosis. Thorax 2011; 66: 624-629.

5 Stoltz DA, Meyerholz DK, Welsh MJ. Origins of cystic fibrosis lung disease. N Engl J Med 2015; 372 : $1574-1575$.

6 Mansell A, Dubrawsky C, Levison H, et al. Lung elastic recoil in cystic fibrosis. Am Rev Respir Dis 1974; 109: 190-197.

7 Soni R, Dobbin CJ, Milross MA, et al. Gas exchange in stable patients with moderate-to-severe lung disease from cystic fibrosis. J Cyst Fibros 2008; 7: 285-291.

8 Stenbit AE, Flume PA. Pulmonary exacerbations in cystic fibrosis. Curr Opin Pulm Med 2011; 17: 442-447.

9 Bhatt JM. Treatment of pulmonary exacerbations in cystic fibrosis. Eur Respir Rev 2013; 22: $205-216$.

10 Hartl D, Gaggar A, Bruscia E, et al. Innate immunity in cystic fibrosis lung disease. J Cyst Fibros 2012; 11: 363-382.

11 Griese M, Kappler M, Gaggar A, et al. Inhibition of airway proteases in cystic fibrosis lung disease. Eur Respir J 2008; 32: 783-795

12 Gaggar A, Li Y, Weathington N, et al. Matrix metalloprotease-9 dysregulation in lower airway secretions of cystic fibrosis patients. Am I Physiol Lung Cell Mol Physiol 2007; 293: L96-L104.

13 Greenlee KJ, Werb Z, Kheradmand F. Matrix metalloproteinases in lung: multiple, multifarious, and multifaceted. Physiol Rev 2007; 87: 69-98.

14 Gaggar A, Hector A, Bratcher PE, et al. The role of matrix metalloproteinases in cystic fibrosis lung disease. Eur Respir J 2011; 38: 721-727.

15 Gaggar A, Jackson PL, Noerager BD, et al. A novel proteolytic cascade generates an extracellular matrix-derived chemoattractant in chronic neutrophilic inflammation. J Immunol 2008; 180: 5662-5669.

16 Vandenbroucke RE, Dejonckheere E, Libert C. A therapeutic role for matrix metalloproteinase inhibitors in lung diseases? Eur Respir J 2011; 38: 1200-1214.

17 Greenwald RA, Moak SA, Ramamurthy NS, et al. Tetracyclines suppress matrix metalloproteinase activity in adjuvant arthritis and in combination with flurbiprofen, ameliorate bone damage. J Rheumatol 1992; 19: 927-938.

18 Golub LM, Sorsa T, Lee HM, et al. Doxycycline inhibits neutrophil (PMN)-type matrix metalloproteinases in human adult periodontitis gingiva. J Clin Periodontol 1995; 22: 100-109.

19 Hanemaaijer R, Visser H, Koolwijk P, et al. Inhibition of MMP synthesis by doxycycline and chemically modified tetracyclines (CMTs) in human endothelial cells. Adv Dent Res 1998; 12: 114-118.

20 Li DQ, Lokeshwar BL, Solomon A, et al. Regulation of MMP-9 production by human corneal epithelial cells. Exp Eye Res 2001; 73: 449-459.

21 Brusasco V, Crapo R, Viegi G, et al. Coming together: the ATS/ERS consensus on clinical pulmonary function testing. Eur Respir J 2005; 26: 1-2.

22 Dasenbrook EC, Checkley W, Merlo CA, et al. Association between respiratory tract methicillin-resistant Staphylococcus aureus and survival in cystic fibrosis. JAMA 2010; 303: 2386-2392.

23 Beringer PM, Owens H, Nguyen A, et al. Pharmacokinetics of doxycycline in adults with cystic fibrosis. Antimicrob Agents Chemother 2012; 56: 70-74.

24 Kim HS, Luo L, Pflugfelder SC, et al. Doxycycline inhibits TGF- $\beta 1$-induced MMP-9 via Smad and MAPK pathways in human corneal epithelial cells. Invest Ophthalmol Vis Sci 2005; 46: 840-848.

25 Amsden GW. Anti-inflammatory effects of macrolides - an underappreciated benefit in the treatment of community-acquired respiratory tract infections and chronic inflammatory pulmonary conditions? J Antimicrob Chemother 2005; 55: 10-21.

26 Wang C, Li D, Qian Y, et al. Increased matrix metalloproteinase-9 activity and mRNA expression in lung injury following cardiopulmonary bypass. Lab Invest 2012; 92: 910-916.

27 Weathington NM, van Houwelingen AH, Noerager BD, et al. A novel peptide CXCR ligand derived from extracellular matrix degradation during airway inflammation. Nat Med 2006; 12: 317-323.

$28 \mathrm{Xu} \mathrm{X}$, Jackson PL, Tanner S, et al. A self-propagating matrix metalloprotease-9 (MMP-9) dependent cycle of chronic neutrophilic inflammation. PloS One 2011; 6: e15781.

29 Malik M, Bakshi CS, McCabe K, et al. Matrix metalloproteinase 9 activity enhances host susceptibility to pulmonary infection with type A and B strains of Francisella tularensis. J Immunol 2007; 178: 1013-1020.

30 Moon A, Gil S, Gill SE, et al. Doxycycline impairs neutrophil migration to the airspaces of the lung in mice exposed to intratracheal lipopolysaccharide. J Inflamm (Lond) 2012; 9: 31.

31 Sommer JU, Schultz JD, Grossbaier J, et al. In vitro effects of doxycycline on inflammatory cytokines and gelatinases in chronic rhinosinusitis. In Vivo 2012; 26: 369-374.

32 Sjowall J, Ledel A, Ernerudh J, et al. Doxycycline-mediated effects on persistent symptoms and systemic cytokine responses post-neuroborreliosis: a randomized, prospective, cross-over study. BMC Infect Dis 2012; 12 : 186.

33 Schulze CJ, Castro MM, Kandasamy AD, et al. Doxycycline reduces cardiac matrix metalloproteinase-2 activity but does not ameliorate myocardial dysfunction during reperfusion in coronary artery bypass patients undergoing cardiopulmonary bypass. Crit Care Med 2013; 41: 2512-2520.

34 Fuchs HJ, Borowitz DS, Christiansen DH, et al. Effect of aerosolized recombinant human DNase on exacerbations of respiratory symptoms and on pulmonary function in patients with cystic fibrosis. The Pulmozyme Study Group. N Engl J Med 1994; 331: 637-642.

35 Elkins MR, Robinson M, Rose BR, et al. A controlled trial of long-term inhaled hypertonic saline in patients with cystic fibrosis. N Engl J Med 2006; 354: 229-240. 
36 Saiman L, Marshall BC, Mayer-Hamblett N, et al. Azithromycin in patients with cystic fibrosis chronically infected with Pseudomonas aeruginosa: a randomized controlled trial. JAMA 2003; 290: 1749-1756.

37 Boyle MP, Bell SC, Konstan MW, et al. A CFTR corrector (lumacaftor) and a CFTR potentiator (ivacaftor) for treatment of patients with cystic fibrosis who have a phe508del CFTR mutation: a phase 2 randomised controlled trial. Lancet Respir Med 2014; 2: 527-538. 